\title{
Non-alcoholic fatty liver diseases: current challenges and future directions
}

\section{Elke Roeb^}

Justus-Liebig-University Giessen, University Hospital UKGM, Giessen, Germany

Correspondence to: Univ.-Prof. Dr. med. Elke Roeb. Head of Gastroenterology, Justus-Liebig-University Giessen, University Hospital UKGM, Klinikstrasse 33, 35392 Giessen, Germany. Email: Elke.Roeb@innere.med.uni-giessen.de.

\begin{abstract}
Non-alcoholic fatty liver diseases (NAFLD) is rapidly becoming the most common cause of chronic liver disease in Western Countries, and a similar trend is expected in Eastern Countries within the next years. This review focusses on the definition of NAFLD and NASH, possible screening mechanisms and the question who should be screened. Still there is a need for non-invasive diagnostic tools and biomarkers for NASH that can quickly and easily diagnose the severity of NAFLD, monitor liver changes, and identify high risk patients. In addition, treatment strategies are discussed as well as the clientele, who should be treated. There are currently no drugs approved for NAFLD. Successful clinical studies with e.g., obeticholic acid and new substances (e.g., cenicriviroc with anti-inflammatory activity) have already been published. If weight-reducing diets and a change in lifestyle fail in the case of severe obesity, bariatric surgery (e.g., gastric bypass or stomach reduction) should be considered. In the case of manifest type 2 diabetes, metformin can be used as an oral antidiabetic of first choice, and GLP-1 agonists have shown beneficial effects on NAFLD. However, up to now the prevention of overweight and lack of exercise targets the most important risk factors. This review aims to identify therapy relevant risk factors, management strategies, and open questions concerning NAFLD patients.
\end{abstract}

Keywords: Non-alcoholic fatty liver diseases (NAFLD); NASH; liver; diabetes; metabolic

Submitted May 06, 2020. Accepted for publication Aug 17, 2020.

doi: 10.21037/atm-20-3760

View this article at: http://dx.doi.org/10.21037/atm-20-3760

"An oversized goose liver, no matter how good it tastes, always requires a sick goose." (Clemens Brentano, 1778-1842).

\section{Introduction}

Non-alcoholic fatty liver diseases (NAFLD) affect about a quarter of the total population in industrialized countries. NAFLD is closely related to demographic changes (aging of society), unhealthy lifestyle (overeating, lack of exercise) and cardiovascular-metabolic diseases (type 2 diabetes, arterial hypertension, dyslipidemia). Very fast, NAFLD became the most common cause of chronic liver disease in Western Countries. We expect a similar trend in Eastern
Countries over the next few years $(1,2)$. The SHIP study (Study of Health in Pomerania) calculated a NAFLD prevalence of approximately $30 \%$ for the age group between 20 and 79 years (3). The range of NAFLD includes nonalcoholic pure (bland) fatty liver (NAFL), steatohepatitis (so called NASH), NASH fibrosis, and liver cirrhosis (NASH cirrhosis) with its associated complications.

By now NAFLD has an estimated prevalence in the Western world of around 25\% (4). About $20 \%$ of these patients experience NASH (5). The progression of NASH is linked to liver cell stress, consecutive inflammation and fibrosis. Furthermore, the development of cirrhosis with portal hypertension and end stage liver disease is possible. In addition, NASH is an important risk factor with regard

$\wedge$ ORCID: 0000-0001-5739-5400. 


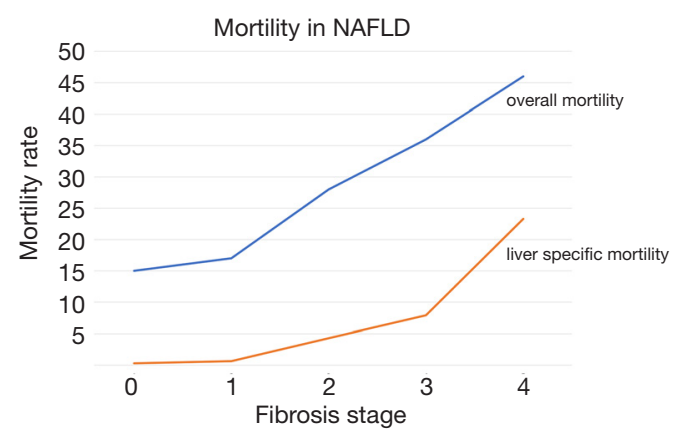

Figure 1 All cause mortality and liver related mortality in NAFLD according to Dulai et al. 2017. Mortality rates are plotted against the stage of fibrosis in all 17,452 patients included in the metaanalysis by Dulai et al. (11). Mortality rate per 1,000 years of patient follow up; fibrosis stage [0-4]. Blue: all over mortality (all causes); red: liver specific mortality.

to hepatocellular carcinoma (HCC). End-stage NASHassociated liver disease is expected to represent the highest proportion of patients who will be listed for liver transplant in the future (6). The disease-although genetic factors have also been identified (7) - is seen as the result of hyperalimentation and as a hepatic manifestation of the socalled metabolic syndrome (8).

In addition to the exclusion of other specific liver diseases, typical clinical, imaging and/or histological characteristics are required for the correct diagnosis. The latter include steatosis, hepatocellular ballooning, lobular inflammation and fibrosis (9). The clinical symptoms of non-cirrhotic NAFLD are usually non-specific. Awareness of the disease and the associated risks are also low (10). The pathogenesis and the natural course of NAFLD are becoming increasingly better understood, even if the heterogeneity of the patients and the multifactorial genesis make it difficult to estimate the individual prognosis. The risk of progression to complications of liver disease (cirrhosis, portal hypertension, liver cancer) is strongly related to the degree of fibrosis. In several prospective observational studies, the degree of fibrosis was associated with total and liver-related lethality $(11,12)$.

A further increase in NAFLD in the sense of the epidemic of obesity, especially among adolescents and younger patients, can be expected. NAFL is present in $15-20 \%$ of the normal population, NASH is observed in $2-3 \%(13)$. The figures for obesity and diabetes are correspondingly higher: $50-75 \%$ have NAFL, $20-50 \%$ have
NASH and 2-3\% already have cirrhosis of the liver (14). Overall and liver related mortality are shown in Figure 1.

According to the study "The heavy burden of obesityThe Economics of prevention" in 34 OECD countries, more than half of the population are already overweight, and one in four is pathologically overweight (15). Between 2010 and 2016, the proportion of obese adults in the OECD countries rose from $21 \%$ to $24 \%$-this corresponds to 50 million people. Just under one in four adults in Germany is obese, in Austria and Switzerland about one in five. Overweight children are particularly hard hit: they do worse at school, have higher absenteeism and less chances of a higher education. They are also bullied up to three times more often than non-obese, which may contribute to their poor school performance (15).

The clinical implications of hepatic steatosis run the risk of being underestimated in several ways. This concerns both their possible causes and the variety of the disease spectrum, the (differential) diagnostic pitfalls, the possible prognostic implications as well as new therapy options and perspectives that might be necessary to manage this widespread disease.

Changes in lifestyle, demographic change and increasing complexity of pharmacological therapies lead to an increase in NAFLD. Doctors and patient organizations have to deal with this collectively and individually. The first S2k guideline for NAFLD at all, the German guideline for NAFLD from 2015 expired in February 2020 and is currently being updated (16). Still there exist a lot of open questions, especially concerning drug therapy and screening. The aim of this review is to better cope with the increased challenges to get on with NAFLD, that is identifying therapy relevant risk factors, management strategies, and open questions.

\section{What is the optimal definition of NAFLD and NASH?}

NAFLD covers a spectrum of liver diseases, which are characterized by increased fat storage in liver cells. The illness includes sole steatosis (NAFL, that is NAFLD), as well as NAFLD hepatitis (nonalcoholic steatohepatitis, NASH), (advanced) NASH fibrosis, cirrhosis, and HCC (17). Between pediatric and adult NAFLD patients there are specific differences in etiology, epidemiology and pathology (18). Diagnostic criteria for NAFLD according to current guidelines include hepatic steatosis verified by imaging or histology $(>5 \%)$, the exclusion of other causes of fatty liver including alcohol, and in some cases the 
existence of insulin resistance (16,19-21). The commonly used acronym is NAFLD (from nonalcoholic fatty liver disease).

Fatty liver or fatty liver hepatitis can be triggered by numerous diseases or causes, e.g., alcoholic liver disease, medication or chemotherapy, hepatitis $\mathrm{C}$ virus infection, fat metabolism disorders or malnutrition. These differential diagnoses should be taken into account as there are specific therapeutic consequences for these different etiologies (16).

Alcoholic fatty liver (AFL) and alcoholic fatty liver hepatitis (ASH) are caused by chronic alcohol abuse. In this context heavy alcohol use implies $>3$ drinks per day in men and $>2$ drinks in women according to the recent ACG guideline (22). In the US, a beverage containing about $14 \mathrm{~g}$ of alcohol equates to one drink, which correlates to 12 ounces of beer ( $5 \%$ weight/volume) or 5 ounces of wine (8-10\% weight/volume), or 1.5 ounces of hard liquor (40$45 \%$ weight/volume) (22).

According to current guidelines NAFLD is defined as steatotic hepatis without significant alcohol consumption and without causes of secondary steatosis. By definition, NAFL and NASH are not caused by alcohol consumption. However, since only about 8 percent of the population between the ages of 18 and 59 live abstinent from alcohol (23), the diagnosis of NAFL and NASH depends on the level of alcohol consumption. In the literature, the threshold of significant alcohol consumption is assumed to be $20-40 \mathrm{~g}$ per day in men and 10-30 g per day in women (24). The current guidelines of EASL and AASLD assume significant alcohol consumption with daily consumption of more than $30 \mathrm{~g}$ for men and more than $20 \mathrm{~g}$ for women. Earlier studies had suggested that daily alcohol consumption of more than $60 \mathrm{~g}$ in men and more than $30 \mathrm{~g}$ in women per se can lead to fatty liver (25). Besides the difficulties in the correct assessment of alcohol intake recent studies show that low to moderate alcohol consumption (in contrast to a higher consumption) is more likely to be associated with a risk reduction for fatty liver (26-28). This evidence (from retrospective data) is still inconclusive because some recently published studies showed that modest alcohol consumption increases hepatic fat without increasing the risk of advanced fibrosis. Prospective data, actually suggest that NAFLD patients with regular alcohol intake, although within the safe thresholds, are at higher risk of liver disease progression, including HCC (29).

The authors of the German guideline recommend distinguishing NAFLD from ALD or mixed forms, with a daily alcohol limit of $10 \mathrm{~g}$ for women and $20 \mathrm{~g}$ for men (16). According to many experimental studies the combination of different unfavorable influences like alcohol, overweight, and free fatty acids, together leads to common damage on hepatocellular lipid accumulation, induces hepatic inflammation, fibrosis and carcinogenesis. Alcohol in rather low dosis already provokes synergistic pathological effects in combination with obesity (30).

There is significant overlap in the pathways by which NAFLD and ALD cause disease. Some data imply an association between drinking of fewer alcohol and reduced $\mathrm{NASH}$ and fibrosis. These studies, however, suffer from significant limitations, which are discussed in detail elsewhere (31). The authors think that there is insufficient evidence for or against moderate alcohol consumption in NAFLD. It is, however, important that NASH as well as advanced fibrosis, delineate hepatic damage including an increase in the risk of HCC, which argues against a moderate alcohol consumption (31). Taken together, counseling NAFLD patients for alcohol abstinence should be maintained.

Combinations of adverse conditions in lifestyle increased the risk for fatty liver, as identified by recently developed predictor algorithm FLI (that is fatty liver index; an algorithm based on waist circumference, body mass index (BMI), triglyceride, and gamma-glutamyltransferase (GGT). The most striking individual impacts on the likelihood for FLI above $60 \%$ were observed for physical inactivity $(\mathrm{P}<0.0005$ for both genders $)$ and alcohol consumption $(\mathrm{P}<0.0005$ for men) $(9,19)$.

Taken together NAFLD is per definition the occurrence of hepatic steatosis (fat in the liver) proven by imaging or liver histology. Secondary causes of hepatic fat accumulation (e.g., increased alcohol consumption, certain medications, and other medical conditions) have to be excluded. Risk factors for fatty liver (NAFL, hepatic steatosis) are summarized in Table 1.

\section{Who should be screened for the presence of NAFLD and how should the screening be carried out?}

A systematic review showed incidence rates of 31 and 86 per 1,000 person-years based on two Japanese papers, but at the same time a significantly lower incidence rate of 29 cases per 100,000 person-years was reported in an English paper (14). Due to the heterogeneous results, further studies are required in order to make more 
Table 1 Risk factors for NAFLD [according to $(16,21,32)$ ]

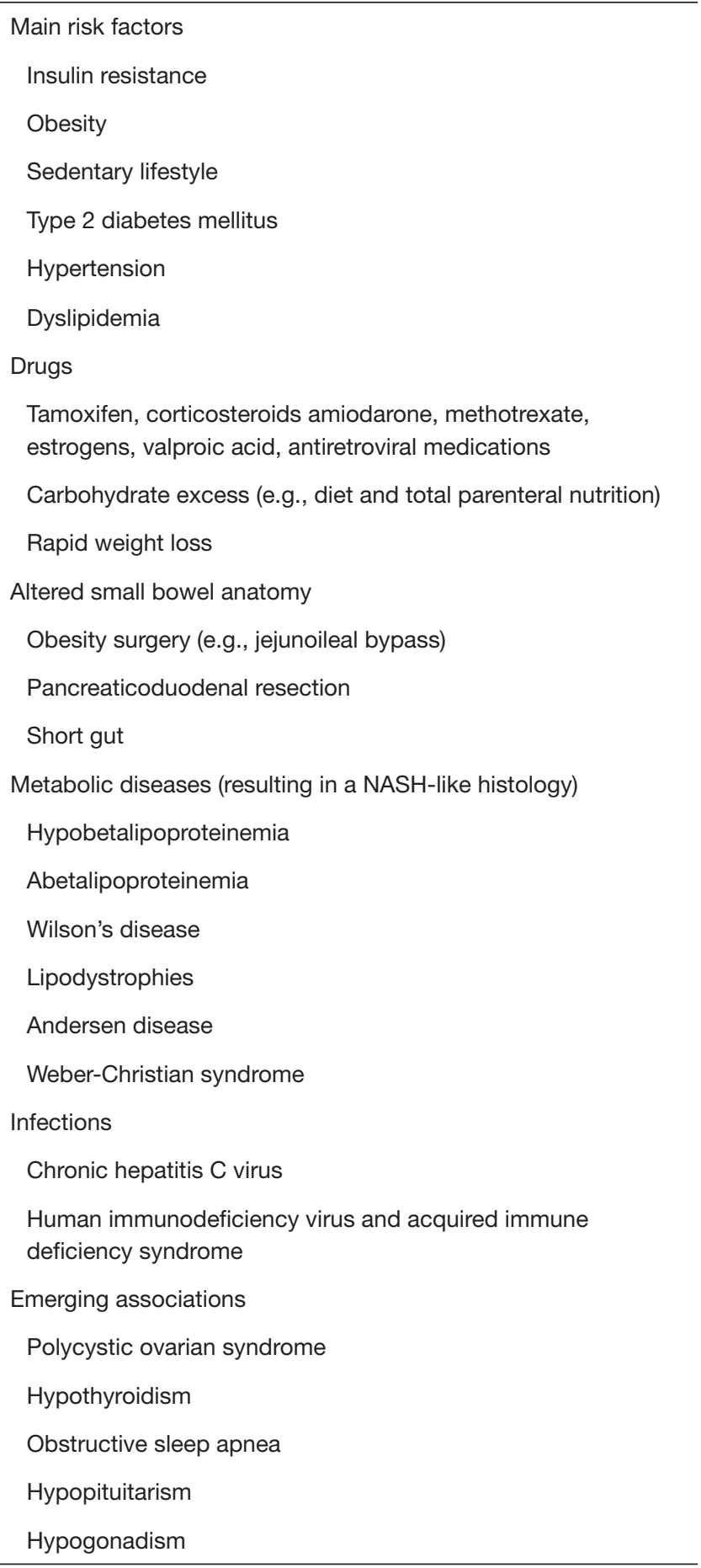

NAFLD, nonalcoholic fatty liver disease.

precise statements about the incidence of NAFLD. Thus, screening programs should be site specific and incorporate the genetic background. Hispanics and Asians are usually less obese but at higher risk than African Americans, who exhibit a lower degree of NAFLD and a lower NAFLD prevalence.

Prevalent NAFLD cases will increase to about $21 \%$, from 83.1 million [2015] to 100.9 million [2030], while prevalent NASH cases will rise to $63 \%$, from 16.52 million to 27.00 million cases (33). These estimates have shown 28,200 liver-related deaths among the NASH population ( $7.6 \%$ of total deaths) in 2015 , increasing to 78,300 or $10.9 \%$ of total deaths in 2030 (33). These are already threatening data especially. Screening programs should be adapted to such high number of entities published recently. Incidence and prevalence are increasing especially in risk populations. Estimated data however have to be proven.

A current study indicates a prevalence of fatty liver of $42.2 \%$ (34). Markus et al. (3) calculated a (similar) prevalence of $39.7 \%$ for the population between 45 and 81 years in Pomerania, northeast of Germany. In the age group between 20 and 79 years, the prevalence of fatty liver was already $29.9 \%$. Overall, the analyzes showed that participants with fatty liver tend to be male, inactive, and smokers.

Among the NAFLD entities, not NAFL but NASH proceeds to cirrhosis and HCC. The epidemic of the overweight and its impact on the increasing prevalence of NAFLD, suggests NASH to be the most common cause of advanced liver disease in coming decades (14).

Musso and coworkers depicted that NAFLD justifies a screening for cardio-metabolic risk and for progressive liver disease. The combination of three noninvasive tests (NAFLD fibrosis score, Cytokeratin 18, elastography) with liver biopsy may optimally individuate patients with NASH, with or without advanced fibrosis (35).

Already in 2007 and 2010, studies by Ruiz and Alisi showed that obese patients with NAFLD have significantly higher endotoxin and LPS levels as well as significantly higher TNF-expression levels in the liver (36,37). However, an increased endotoxin content was not found in a pediatric NASH cohort (38).

Depending on the patients' predisposition, lipid accumulation could promote lipotoxicity and mitochondrial dysfunction, thus triggering hepatocyte inflammation, death, and fibrosis. In this context free fatty acids and free cholesterol have been ascertained to be toxic. To make the picture more complex, the pathogenesis of NAFLD encompasses pathological connections between the liver and other organs, including the adipose tissue and the gut (39). Nonetheless, presently there is no accurate noninvasive method to detect NASH. 
Laboratory screening by means of the determination of transaminases is not sensibly possible, since patients with NAFLD predominantly have normal transaminases (40). The prevalence of ultrasound-detected steatosis in the general population is on average around 30\% high (14), but the sensitivity of ultrasound for the detection of steatosis is relatively low at around $60 \%$ for a screening procedure (41). In addition, sonography is expensive and requires more resources and expertise.

The prevalence of NAFLD (defined as liver fat concentration $\geq 6 \%$ ) was low in type 1 diabetes $(8.8 \%)$ but high in type 2 diabetes (T2D). In a recently published single center study with T2D patients, NAFLD was associated with several markers of insulin resistance (42). Lai et al. screened for NAFLD and advanced fibrosis among patients suffering from type 2 diabetes. The majority of these 560 patients had non-alcoholic steatohepatitis (about $83 \%)$ and $\geq \mathrm{F} 1$ fibrosis (87\%) while advanced fibrosis was seen in $37 \%$ (43). Thus, the prevalence of NAFLD and advanced fibrosis identified by transient elastography is rather high among T2DM patients.

There are some arguments in favor of considering screening in high-risk groups such as diabetics and obese people, in whom NAFLD has a prevalence of up to $70 \%$ (44). Missing data with regard to diagnostic criteria, prediction of the course and progress as well as established therapy indications and procedures do not currently allow a recommendation for a screening.

Screening of overweight and obese children in primary care for NAFLD with referral to paediatric gastroenterology has the potential to identify clinically relevant liver pathology (45). However, many children with suspected NAFLD were shown to have liver disease other than NAFLD. Elevation of transaminases was combined with worsening of the disease. Levels of ALT, however, could not discriminate between stages of NAFLD on the individual patient level. Importantly, the screening and referral process followed by liver biopsy was able to identify many obese children with advanced fibrosis that would have otherwise remained undiagnosed. Amongst children with NAFLD, approximately half had steatohepatitis (45).

A liver biopsy is the method of choice to determine the stage of fibrosis. However, due to the risk of complications and the high costs, this screening method is not suitable for use within the general population. Hagström et al. have now examined the performance of 5 non-invasive scoring systems for the prediction of severe liver diseases. The authors collected data from the Swedish Apolipoprotein
Mortality Risk cohort on persons 35 to 79 years old from 1985 through 1996. APRI ( $\mathrm{n}=127,302)$, BARD $(\mathrm{n}=75,303)$, FIB-4 ( $n=126,941)$, Forns $(n=122,419)$, and the nonalcoholic fatty liver disease (NAFLD) fibrosis score (NFS, $n=13,160$ ) were applied. The predictive values of the scoring systems applied decreased with time and were more meaningful in men. All scoring systems were more accurate in attendees with risk factors for NAFLD at baseline, with AUROCs reaching 0.83 . The predictive ability was modest. Finally, there is a need for better scoring systems to evaluate the risk of fibrosis in the total population (46).

Lean NAFLD is drawing considerable attention. Leung et al. prospectively followed up 72 lean (BMI $<25 \mathrm{~kg} / \mathrm{m}^{2}$ ) and 235 overweight (BMI $\geq 25 \mathrm{~kg} / \mathrm{m}^{2}$ ) patients with biopsy-proven NAFLD. Lean patients had a lower grade of steatosis and lower stage of fibrosis than overweight patients. In addition, the event-free survival was better in lean patients $(47,48)$.

Taken together screening for NAFLD in adults is currently not recommended for the general population (16). For risk groups, examinations for the existence of a NAFLD can be carried out. Patients with NAFLD who either have clinical symptoms or signs of liver disease or abnormal liver-associated laboratory values should be evaluated for the presence of NASH.

NAFLD patients who have neither clinical symptoms nor signs of liver disease or abnormal liver-associated laboratory values should be evaluated for the presence of cardio-metabolic diseases, alcohol consumption and drug side effects.

Not to determine NAFL but to determine a severe form of NAFLD (NASH, NASH fibrosis, NASH cirrhosis) is necessary to define the associated prognosis. Presence and stage of fibrosis, which is the most important determinant for the severity and progression of NAFLD should be confirmed.

\section{What is an ideal and economical diagnostic tool?}

Still, there is a generally accepted need for non-invasive diagnostic tools and biomarkers for NASH and fibrosis that can quickly and easily diagnose the severity of NAFLD, monitor liver changes, and identify high risk patients (21).

Modern biomarkers include prediction models (e.g., NAFLD fibrosis score, FIB-4 index and BARD score) or detection of inflammation (e.g., circulating keratin 18 fragments), or fibrosis (e.g., FibroTest ${ }^{\circledR}$, ELF $^{\mathrm{TM}}$ or 
Pro-C3 tests). For clinical use, biomarkers may discriminate between patients with NASH or advanced fibrosis, predict dynamic changes in NASH/fibrosis over time, and provide long-term prognostic information (12).

NAFLD fibrosis score (NFS) as well as FIB-4 index seem to be both, cost-effective and very sensitive tools to identify patients with advanced fibrosis. Fibrosis scores for NAFLD are summarized in Table 2.

Srivastava and colleagues investigated a diagnostic algorithm for NAFLD patients, in order to detect severe fibrosis and cirrhosis. By conducting a prospective cohort study, they analysed whether the sequential use of FIB-4 and ELF in over 3,000 patients reduced avoidable referrals from primary care to secondary care by $81 \%$ (50).

By combining clinical risk factors (dangerous alcohol consumption, elevated transaminases, risk factors of the metabolic syndrome), serum markers, and transient elastography primary care programs were able to identify more patients with advanced liver disease in alcoholic as well as in non-alcoholic liver diseases (51).

Methods have been developed, that detect liver fat (NAFL) using magnetic resonance imaging-derived proton density fat fraction (MRI-PDFF) on routine MRI devices and do not require any additional spectroscopy coils (52). MRI-PDFF is based on the indirect estimate of accompanying changes in fatty liver (T1 deviation, T2 deviation and T2 drop, spectral complexity of fat, deviation when integrating separately acquired water and fat images into a combined image to record the fat fraction), but not of significant influencing factors of the NAFLD, e.g., body mass index, inflammation or iron overload. The etiology of liver disease also has no influence on the detection of fat content. In recent years, MRI-PDFF has shown high diagnostic accuracy with the histological degree of steatosis. It is now recognized as a reference standard in diagnostic radiology (53). So far, results of noninvasive screening procedures are suboptimal. However, MRI-PDFF presents the most accurate modality for detecting hepatic fat or NAFL. On the other side MRE appears to be an accurate method for scaling liver disease. MRI-PDFF in combination with MRE might provide a precise method for stratifying NAFLD patients with regard to their overall risks. Still, total costs and long waiting times for these modern techniques display a major challenge to most physicians and hospitals.

Liver biopsy still remains the current gold standard for diagnosis, despite limitations regarding sampling variability, invasive nature, and high cost (16). Currently, liver biopsy is also considered the gold standard for acquisition of NASH and determination of the fibrosis stage. On the other hand, tissue biopsy includes a high standard deviation with regard to characteristic features of NASH. Kose $e t$ al. did not record severe adverse events (e.g., fever, abscessing, anaphylactic shock, organ damage, sepsis, deaths). Despite a certain invasiveness, they considered percutaneous liver biopsy as being safe and with low complications (54). Up to now liver biopsy is the only diagnostic that can clearly differentiate between NAFL, NAFL with low inflammation and NASH (steatosis with lobular and portal inflammation and hepatocellular ballooning), and defining the presence or absence of even low fibrosis (21). For clinical purposes, a simple but robust algorithm for categorizing liver lesions in NAFLD patients was introduced by Bedossa et al. (55). Nevertheless, severe complications and fulminant internal haemorrhage are discussed in patient consent forms for sonography guided percutaneous liver biopsy.

Ultrasound-based transient hepatic elastography has been the first true bedside technique to reproducible screen for liver fibrosis. In comparison to other techniques TE has an excellent interobserver variability, small sampling error, and good reproducibility. If liver fibrosis is suspected, TE should be performed directly after the abdominal ultrasound and routine blood tests. In cases of severe obesity (BMI >30) or ascites, the XL probe should be used (56).

There are no scientific data on risk adaptation or costeffectiveness of NAFLD diagnostics. Step diagnostics takes parameters into account based on their availability, costs/ effort and pretest probability of positive results. Due to the high prevalence of NAFLD, the implementation of a level 1 for the identification of high-risk patients (NASH, NASH fibrosis) who should be further diagnosed, appears to be of particular importance. Studies indicate that this can be improved further despite medical knowledge of the relevance of NAFLD.

In addition, cytokeratin 18 fragments in the blood, also called M30, represent novel biomarkers for the presence of inflammation and apoptosis in NASH. So far circulating cytokeratin 18 fragments are not recommended by guidelines. Noninvasive tests are needed. A better stratification of the patients could for example be achieved by a combination of different markers, e.g., NFS with M30 or NFS with TE, or all three markers together to ensure an accurate identification of high-risk patients with advanced inflammation and fibrosis (57).

Taken together NAFL in most cases is diagnosed initially by ultrasound, quantified by CAP in daily routine and by 
Table 2 Fibrosis scores appropriate for NAFLD [according to $(16,19,21,32,49)$ and literature indicated in the right column]

\begin{tabular}{|c|c|c|c|}
\hline Score & Components & Risk of fibrosis & Literature \\
\hline $\begin{array}{l}\text { NFS, NAFLD } \\
\text { fibrosis score }\end{array}$ & $\begin{array}{l}\text { Serum glucose, platelet count, } \\
\text { albumin, AST/ALT ratio; } \\
\text { age, BMI, and diabetes status }\end{array}$ & $\begin{array}{l}>0.676 \text { (fibrosis), } \\
<-1.455 \text { (no } \\
\text { fibrosis) }\end{array}$ & $\begin{array}{l}\text { Angulo P, Hui JM, Marchesini G, et al. The NAFLD } \\
\text { fibrosis score: a noninvasive system that identifies } \\
\text { liver fibrosis in patients with NAFLD. Hepatology } \\
2007 ; 45: 846-54\end{array}$ \\
\hline BARD & BMI, diabetes, AST/ALT ratio & $\begin{array}{l}0-1 \text { : low risk, } 2-4 \text { : } \\
\text { high risk, NPV } \\
97 \%\end{array}$ & $\begin{array}{l}\text { Harrison SA, Oliver D, Arnold HL, et al. Development } \\
\text { and validation of a simple NAFLD clinical scoring } \\
\text { system for identifying patients without advanced } \\
\text { disease. Gut 2008;57:1441-7 }\end{array}$ \\
\hline $\begin{array}{l}\text { ELF }^{\circledR} \text { enhanced } \\
\text { liver fibrosis test } \\
\text { commercially } \\
\text { available }\end{array}$ & $\begin{array}{l}\text { Hyaluronic acid }(\mathrm{HA}) \text {, procollagen } \\
\text { III amino-terminal peptide (PIIINP), } \\
\text { and tissue inhibitor of matrix } \\
\text { metalloproteinase } 1 \text { (TIMP-1) }\end{array}$ & $\begin{array}{l}>10.51 \text { advanced } \\
\text { fibrosis }\end{array}$ & $\begin{array}{l}\text { https://www.nice.org.uk/guidance/ng49/chapter/ } \\
\text { recommendations }\end{array}$ \\
\hline $\begin{array}{l}\text { Pro-C }{ }^{\circledR} \text { test } \\
\text { commercially } \\
\text { available }\end{array}$ & $\begin{array}{l}\text { NASH biomarker synthesis of type } \\
\text { III collagen, propeptide }\end{array}$ & & $\begin{array}{l}\text { Gloomba R, Sanyal AJ. The global NAFLD epidemic. } \\
\text { Nat Rev Gastroenterol Hepatol 2013;10:686-90. www. } \\
\text { bms.com/researchers-and-partners/areas-of-focus. } \\
\text { html }\end{array}$ \\
\hline \multicolumn{4}{|l|}{ Imaging methods } \\
\hline $\begin{array}{l}\text { FibroScan }^{\circledR} \\
\text { transient hepatic } \\
\text { elastography }\end{array}$ & Liver stiffness measurements & $\begin{array}{l}<7 \mathrm{kPa} \text { normal, } \\
<12 \mathrm{kPa} \text { severe } \\
\text { fibrosis }\end{array}$ & $\begin{array}{l}\text { Sandrin L, Fourquet B, Hasquenoph JM, et al. } \\
\text { Transient elastography: a new noninvasive method for } \\
\text { assessment of hepatic fibrosis. Ultrasound Med Biol } \\
\text { 2003;29:1705-13 }\end{array}$ \\
\hline \multicolumn{4}{|l|}{$\begin{array}{l}\text { Dynamic liver } \\
\text { function test }\end{array}$} \\
\hline $\begin{array}{l}\text { LiMAx (liver } \\
\text { maximum capacity) }\end{array}$ & $\begin{array}{l}\text { Reflects cytochrome P450 1A2 } \\
\text { activity }\end{array}$ & $\begin{array}{l}\text { LiMAx values } \\
>315 \mu \mathrm{g} / \mathrm{kg} / \mathrm{h} \text { are } \\
\text { considered normal }\end{array}$ & $\begin{array}{l}\text { Blüthner E, Pape UF, Stockmann M, et al. Assessing } \\
\text { Non-Invasive Liver Function in Patients with Intestinal } \\
\text { Failure Receiving Total Parenteral Nutrition-Results } \\
\text { from the Prospective PN Liver Trial. Nutrients } \\
\text { 2020:26:12 }\end{array}$ \\
\hline
\end{tabular}


MR in studies. Fibrosis is diagnosed by liver elastography. NASH could be determined by biopsy or probably by some serum tests. A recently published noninvasive lipidomic serum test assessed by two panels of triglycerides distinguished between NAFLD and NAFL and between NASH and NAFL with high accuracy (58). Thus, metabolomics provides a new technology for noninvasive biomarkers to improve NAFLD diagnosis.

Diagnostic algorithms for differentiating NAFLD by non-invasive tools, including transient hepatic elastography, are presented by Roeb et al. in $(16,59)$.

\section{How should we treat the patients, who have to be treated at all?}

NAFL displays hepatic steatosis without evidence of inflammation and increased risk of developing liver-related complications. Patients with NASH, however, should be treated to prevent NASH progression to advanced fibrosis, to prevent cirrhosis and to prevent the development of its hepatic complications.

The therapeutic goal or rationale of NAFLD is to reduce insulin resistance with reduced cardiovascular end organ damage, reduced liver failure (including HCC), and the extension of lifetime. The change in lifestyle in terms of an increased physical activity as well as a moderate weight loss is able to delay or even prevent insulin resistance. Regular physical activity reduces the risk of NAFLD. Increased calorie intake, however, increases the corresponding risk (16).

Hepatic fibrosis displays the main characteristic predicting liver related and all cause mortality in NAFLD. Cenicriviroc, a promising new antifibrotic agent, blocks the CCR5 and CCR2 receptor. The efficacy and safety of cenicriviroc in patients with NASH and liver fibrosis were assessed in the CENTAUR study, a phase 2b, double-blind, randomized, placebo-controlled, multinational study with 289 NASH subjects (60). In the Cenicriviroc group, the fibrosis score remained improved by at least one level, with patients with advanced fibrosis benefiting the most (60). A phase 3 trial (NCT03028740) will have to clarify whether the results are stable.

Friedman et al. summarizes the therapeutical targets: metabolic pathways, cell stress and apoptosis, immune targets, fibrosis, and the intestinal microbiome and brown adipose tissue. Thus, sites of drug action-currently in clinical trials-are based on their primary locus of activity. Targets include fatty acids, insulin resistance, immune signals, inflammatory cells caspases, oxidant stress, extracellular matrix, hepatic stellate cells, other key features of fibrogenesis as well as glucose and lipid homeostasis.

There are currently no drugs approved for the NAFLD indication. There are promising phase 3 studies on the FXR agonist obeticholic acid and elafibrinor, a dual $\mathrm{PPAR} \alpha / \delta$ agonist. Metformin, thiazolidines and glucagonlike peptide-1 receptor agonists (GLP-1 RA) represent interesting candidates in order to treat type 2 diabetics with NAFLD (61). The risk of developing type 2 diabetes decreases with the improvement of fatty liver and certain therapies (e.g., pioglitazone) are effective both in the treatment of hyperglycemia and in NAFLD $(21,24,62)$. Numerous substances have been and are being analyzed for their effectiveness in NASH in extensive studies. Without claiming to be complete Roeb \& Geier provide an up-todate overview about the different therapeutic approaches at NAFLD and NASH (63). Furthermore, the combination of compounds that addressed various targets of the metabolic pathways involved in NASH progression, e.g., GLP-RA/ glucagon receptor agonist and GLP-RA/gastrointestinal peptide agonist, are promising future options $(63,64)$. SGLT2 inhibitors as well as GLP-1 RAs might have a positive effect on the treatment of NAFLD in type 2 diabetics. A systematic review was published recently (65).

NASH is a rapidly growing etiology of end-stage liver disease in the US and elsewhere with significantly higher post-transplant survival compared to $\mathrm{HCV}$ (66). Currently $\mathrm{NASH}$ is the second leading cause for liver transplantation overall, even the leading cause in in females. Given the rate of increase, NASH will likely rise to become the leading indication for liver transplantation in males as well $(66,67)$.

Therapy of cardiovascular risks and associated metabolic diseases has to be considered because of mortality data. For each therapeutic strategy monitoring as well as an individualized follow-up of the treatment strategies is mandatory. Up to now NAFLD and NASH are considered as one disease. Since we observe different outcomes the management of non-cirrhotic NAFLD cases should be differentiated from cirrhosis in therapy strategies.

Finally, up to now, treatment of this disease is limited to lifestyle modifications.

\section{What is the minimum therapy? What are essential conservative therapeutic measures?}

The following recommendations are mentioned in nearly all guidelines $(9,16,19,68-70)$ published so far: 
* In overweight or obese patients with NAFLD, regardless of the composition of their macronutrients, a weight-reducing diet change should initially be used with the aim of sustainable weight loss of $0.5-1.0 \mathrm{~kg}$ per week.

* NAFLD patients should be recommended to exercise regularly in the form of endurance and/or strength training.

* Patients with compensated NASH cirrhosis should be motivated to undertake a sports program under supervision.

* In decompensated NASH cirrhosis patients, muscle therapy should be used to counteract muscle loss.

* Patients with NAFLD should be informed about the risks of smoking.

* Patients with NAFLD should refrain from drinking alcohol.

* Patients with NASH cirrhosis should strictly avoid alcohol.

* Drinking of coffee should be recommended due to beneficial effects on the liver and the cardiovascular system.

* An indicated statin therapy can be maintained until liver values increase up to 3 times the upper normal value.

* Metabolically neutral antihypertensives should be given preference in the case of hypertension requiring treatment-primarily inhibitors of the reninangiotensin-aldosterone system.

* With decompensated liver cirrhosis, vasodilating drugs should not be used.

* In the case of manifest type 2 diabetes up to the Child A stage, metformin can be used as an oral antidiabetic drug of choice, even in the case of elevated transaminases.

* Vaccination according to respective national vaccination recommendations (e.g., STIKO-, ECDC, and CDC-guidelines) should be given to patients with chronic liver diseases, especially against hepatitis A, hepatitis B, and influenza (71-73).

* Regular monitoring with the aim of early detection of HCC should be carried out according to the recommendations in the Diagnostics section.

\section{Which pharmacological therapies are permitted?}

Since simple NAFL, unlike NASH, is not associated with over-mortality or liver-associated morbidity, from a hepatological point of view, even in the absence of large randomized studies, drug therapy is only an option for patients with secured NASH (21).

An overview of NAFLD agents currently under development and their pharmacologic targets is provided by Sumida et al. (61). There are 5 candidates who have been successful in studies, but which will be launched in 2021 at the earliest (obeticholic acid, elafibranor, selonsertib, cenicriviroc, and resmetirom) (74). The results of the FLINT study were recently published, in which the administration of obeticholic acid, a farnesoid $\mathrm{X}$ receptor agonist, was associated with a significant improvement in the histological characteristics of NASH including fibrosis compared to placebo (75). A total of $25 \mathrm{mg}$ obeticholic acid significantly improved fibrosis and key components of NASH disease activity among patients with NASH (76). The authors demonstrated histological improvements. The most common adverse event was pruritus $(51 \%)$ in the obeticholic acid $25 \mathrm{mg}$ group. Pruritus incidence however was generally mild to moderate in severity (76).

Cenicriviroc is an inhibitor of CCR2 and CCR5 receptor and initially an experimental drug candidate for the treatment of HIV infection. According to Friedman et al. given the urgent need to develop antifibrotic therapies in NASH, these findings warrant phase 3 evaluation (77). After 1 year of Cenicriviroc treatment, twice as many subjects achieved improvement in fibrosis and no worsening of steatohepatitis compared with placebo (77).

Levels of hepatic aminotransferases could be lowered by administration of vitamin $\mathrm{E}$ and pioglitazone. In addition, both substances improved hepatic steatosis and inflammation. Improvement of fibrosis scores, however, could not be achieved. Thus, the authors concluded that pioglitazone was no better than placebo with regard to the primary outcome (78).

\section{Surgical and endoscopic therapeutical procedures}

Adipo-surgery should be offered to patients with a BMI $\geq 35 \mathrm{~kg} / \mathrm{m}^{2}$ who are suffering from one or more obesityassociated morbidities, e.g., metabolic syndrome with type 2 diabetes, disorders of fat metabolism, coronary heart failure, high blood pressure, NAFLD or NASH, kidney damage, sleep apnea, obesity-hypoventilation syndrome, gastrooesophageal reflux disease, pseudotumor cerebri, bronchial asthma, chronic varicose veins, urinary incontinence, immobilizing joint disease, fertility restrictions or polycystic 
ovarian syndrome, if the conservative therapy is exhaustive.

Patients with a BMI $\geq 40 \mathrm{~kg} / \mathrm{m}^{2}$ and coexistent type 2 diabetes not only benefit from better glycemic control or reduced antidiabetic medication during a metabolic intervention, but also from sustainable weight reduction. This is superior to conservative therapeutic approaches. Evidence of exhausted conservative therapy in the sense of obesity surgery is not required for the indication of the operation in these patients $(9,79,80)$.

The gastric band, tubular stomach formation, proximal Roux-en-Y gastric bypass and omega-loop gastric bypass may be considered as an obesity surgical or metabolic intervention in patients aged 65 or older. Most of the evidence is available for proximal Roux-en- $Y$ gastric bypass and for gastric tube formation (80).

The gastric balloon can be offered to patients with obesity after failure of conservative therapy and if surgery is refused or if there are contraindications to surgery as part of an appropriate accompanying program. The implantation of the Endobarrier ${ }^{\mathrm{TM}}$ can currently only be recommended as part of studies.

There is growing evidence on mutual relationships between NAFLD and type 2 diabetes. For this reason, guidelines including bariatric surgery as well as endoscopic procedures on patients with grade II obesity $(\mathrm{BMI}>35)$ or more are currently being created by scientific organizations such as the National Institute for Health and Care Excellence (NICE) in the UK, EASL, EASD and EASO.

\section{Which monitoring and long-term management is optimal?}

For the prevention of NAFLD, especially in pediatric particularities, positive recommendation for lifestyle changes with weight loss and increased physical activity as well as the refraction from fructose-containing sweetened foods and drinks, especially in the form of snacks, can be given. Intensity of long term monitoring depends on grade of fibrosis and establishment of cirrhosis. For capacity reasons HCC screening every 6 months is reserved for patients with NASH fibrosis and NASH cirrhosis only. Here, reference is made to the corresponding HCC guidelines from AASLD (69) and EASL (70).

\section{Conclusions \& remaining open questions}

Meanwhile, NAFLD has become the most common chronic liver disease in industrialized countries. The prevention of overweight and lack of exercise targets the most important risk factors. Physical activity has been shown to improve fatty liver even without reducing body weight. So far, no specific drug therapy has been approved for this illness, neither for NASH nor for NAFLD in general. Despite diverse publications the following questions have not yet been answered. An intensive discussion is required for the time being:

* What is the optimal definition of NAFLD and NASH?

* How can NASH be diagnosed in primary care?

* Who should be followed up and when?

* What is the minimal therapy?

* What are essential conservative therapeutic measures?

* Which monitoring and long-term management is optimal?

* What is the optimal frequency of follow-up examinations?

\section{Acknowledgments}

Funding: This work was funded by Deutsche Forschungsgemeinschaft Ro 957/8-1, Ro957/11-1, vonBehring-Röntgen Foundation \#66-0008, and UKGM $\# 10 / 2013$.

\section{Footnote}

Provenance and Peer Review: This article was commissioned by the Guest Editors (Ralf Weiskirchen and Wolfgang Stremmel) for the series "Unresolved Basic Issues in Hepatology" published in Annals of Translational Medicine. The article was sent for external peer review organized by the Guest Editors and the editorial office.

Peer Review File: Available at http://dx.doi.org/10.21037/ atm-20-3760

Conflicts of Interest: The author has completed the ICMJE uniform disclosure form (available at http://dx.doi. org/10.21037/atm-20-3760). The series "Unresolved Basic Issues in Hepatology" was commissioned by the editorial office without any funding or sponsorship. The author has no other conflicts of interest to declare.

Ethical Statement: The author is accountable for all aspects of the work in ensuring that questions related to the accuracy or integrity of any part of the work are 
appropriately investigated and resolved.

Open Access Statement: This is an Open Access article distributed in accordance with the Creative Commons Attribution-NonCommercial-NoDerivs 4.0 International License (CC BY-NC-ND 4.0), which permits the noncommercial replication and distribution of the article with the strict proviso that no changes or edits are made and the original work is properly cited (including links to both the formal publication through the relevant DOI and the license). See: https://creativecommons.org/licenses/by-nc-nd/4.0/.

\section{References}

1. Sayiner M, Koenig A, Henry L, et al. Epidemiology of nonalcoholic fatty liver disease and nonalcoholic steatohepatitis in the United States and the rest of the world. Clin Liver Dis 2016;20:205-14.

2. Younossi Z, Tacke F, Arrese M, et al. Global Perspectives on Nonalcoholic Fatty Liver Disease and Nonalcoholic Steatohepatitis. Hepatology 2019;69:2672-82.

3. Markus MRP, Baumeister SE, Stritzke J, et al. Hepatic steatosis is associated with aortic valve sclerosis in the general population: the Study of Health in Pomerania. (SHIP). Arterioscler Thromb Vasc Biol 2013;33:1690-5.

4. Lindor KD, Kowdley KV, Harrison ME, et al. ACG Clinical Guideline: Primary Sclerosing Cholangitis. Am J Gastroenterol 2015;110:646-59.

5. Younossi ZM, Loomba R, Anstee QM, et al. Diagnostic modalities for nonalcoholic fatty liver disease, nonalcoholic steatohepatitis, and associated fibrosis. Hepatology 2018;68:349-60.

6. Cholankeril G, Wong RJ, Hu M et al. Liver Transplantation for non-alcoholic steatohepatitis in the US: Temporal trends and outcomes. Dig Dis Sci 2017;62:2915-22.

7. Eslam M, Valenti L, Romeo S. Genetics and epigenetics of NAFLD and NASH: Clinical impact. J Hepatol 2018;68:268-79.

8. Bellentani S. The epidemiology of non-alcoholic fatty liver disease. Liver Int 2017;37:81-4.

9. European Association for the Study of the Liver (EASL) European Association for the Study of Diabetes (EASD); European Association for the Study of Obesity (EASO). EASL-EASD-EASO Clinical Practice Guidelines for the management of non-alcoholic fatty liver disease. J Hepatol 2016;64:1388-402.</jrn>

10. Sattar N, Forrest E, Preiss D. Non-alcoholic fatty liver disease. BMJ 2014;349:g4596.

11. Dulai PS, Singh S, Patel J, et al. Increased risk of mortality by fibrosis stage in nonalcoholic fatty liver disease: Systematic review and meta-analysis. Hepatology 2017;65:1557-65.

12. Vilar-Gomez E, Calzadilla-Bertot L, Wai-Sun Wong V, et al. Fibrosis severity as a determinant of cause-specific mortality in patients with advanced nonalcoholic fatty liver disease: a multi-national cohort study. Gastroenterology 2018;155:443-57.e17.

13. Blachier M, Leleu H, Peck-Radosavljevic M, et al. The burden of liver disease in Europe. A Review of available epidemiological data. European Association for the Study of the Liver 2013. J Hepatol 2013;58:593-608.

14. Vernon G, Baranova A, Younossi ZM. Systematic review: the epidemiology and natural history of non-alcoholic fatty liver disease and non-alcoholic steatohepatitis in adults. Aliment Pharmacol Ther 2011;34:274-85.

15. OECD (2019), The Heavy Burden of Obesity: The Economics of Prevention, OECD Health Policy Studies, OECD Publishing, Paris. Available online: https://doi. org/10.1787/67450d67-en (6th May 2020)</bok>

16. Roeb E, Steffen HM, Bantel H, et al. S2k Guideline non-alcoholic fatty liver disease. Z Gastroenterol 2015;53:668-723.

17. Brunt EM. Nonalcoholic steatohepatitis: pathologic features and differential diagnosis. Semin Diagn Pathol 2005;22:330-8.

18. Tannapfel A, Denk H, Dienes HP, et al. Histopathological diagnosis of non-alcoholic and alcoholic fatty liver disease. Virchows Arch 2011;458:511-23.

19. European Association for the Study of the Liver (EASL), European Association for the Study of Diabetes (EASD), European Association for the Study of Obesity (EASO): EASL-EASD-EASO Clinical practice guidelines for the management of non-alcoholic fatty liver disease. Diabetologia 2016;59:1121-40.</jrn>

20. Wong VW, Chan WK, Chitturi S, et al. Asia-Pacific Working Party on Non-alcoholic Fatty Liver Disease guidelines 2017-Part 1: Definition, risk factors and assessment. J Gastroenterol Hepatol 2018;33:70-85.

21. Chalasani N, Younossi Z, Lavine JE, et al. The diagnosis and management of nonalcoholic fatty liver disease: Practice guidance from the American Association for the Study of Liver Diseases. Hepatology 2018;67:328-57.

22. Singal AK, Bataller R, Ahn J, et al. ACG Clinical Guideline: Alcoholic Liver Disease. Am J Gastroenterol 2018;113:175-94. 
23. German Center for Addiction Issues. Available online: www.dhs.de (4th May 2020)

24. Sanyal AJ, Brunt EM, Kleiner DE, et al. Endpoints and clinical trial design for nonalcoholic steatohepatitis. Hepatology 2011;54:344-53.

25. Bellentani S, Saccoccio G, Masutti F, et al. Prevalence of and risk factors for hepatic steatosis in Northern Italy. Ann Intern Med 2000;132:112-7.

26. Sookoian S, Castaño GO, Pirola CJ. Modest alcohol consumption decreases the risk of non-alcoholic fatty liver disease: a meta-analysis of 43175 individuals. Gut 2014;63:530-2.

27. Dunn W, Xu R, Schwimmer JB. Modest wine drinking and decreased prevalence of suspected nonalcoholic fatty liver disease. Hepatology 2008;47:1947-54.

28. Dunn W, Sanyal AJ, Brunt EM, et al. Modest alcohol consumption is associated with decreased prevalence of steatohepatitis in patients with non-alcoholic fatty liver disease (NAFLD). J Hepatol 2012;57:384-91.

29. Petroni ML, Brodosi L, Marchignoli F, et al. Moderate Alcohol Intake in Non-Alcoholic Fatty Liver Disease: To Drink or Not to Drink? Nutrients 2019;11:3048.

30. Mahli A, Hellerbrand C. Alcohol and Obesity: A Dangerous Association for Fatty Liver Disease. Dig Dis 2016;34:32-9.

31. Ajmera VH, Terrault NA, Harrison SA. Is moderate alcohol use in nonalcoholic fatty liver disease good or bad? A critical review. Hepatology 2017;65:2090-9.

32. Younossi Z, Anstee QM, Marietti M, et al. Global burden of NAFLD and NASH: trends, predictions, risk factors and prevention. Nat Rev Gastroenterol Hepatol 2018;15:11-20.

33. Estes C, Razavi H, Loomba R, et al. Modeling the epidemic of nonalcoholic fatty liver disease demonstrates an exponential increase in burden of disease. Hepatology 2018;67:123-33.

34. Kühn JP, Meffert P, Heske C, et al. Prevalence of fatty liver disease and hepatic iron over-load in a northeastern German population by using quantitative MR imaging. Radiology 2017;284:706-16.

35. Musso G, Gambino R, Cassader M, et al. Meta-analysis: natural history of non-alcoholic fatty liver disease (NAFLD) and diagnostic accuracy of non-invasive tests for liver disease severity. Ann Med 2011;43:617-49.

36. Ruiz AG, Casafont F, Crespo J et al. Lipopolysaccharidebinding protein plasma levels and liver TNF-alpha gene expression in obese patients: evidence for the potential role of endotoxin in the pathogenesis of non-alcoholic steatohepatitis. Obes Surg 2007;17:1374-80.

37. Alisi A, Manco M, Devito R, et al. Endotoxin and plasminogen activator inhibitor-1 serum levels associated with nonalcoholic steatohepatitis in children. J Pediatr Gastroenterol Nutr 2010;50:645-9.

38. Yuan J, Baker SS, Liu W, et al. Endotoxemia unrequired in the pathogenesis of pediatric nonalcoholic steatohepatitis. J Gastroenterol Hepatol 2014;29:1292-8.

39. Pierantonelli I, Svegliati-Baroni G. Nonalcoholic Fatty Liver Disease: Basic Pathogenetic Mechanisms in the Progression From NAFLD to NASH. Transplantation 2019;103:e1-13.

40. Kotronen A, Westerbacka J, Bergholm R, et al. Liver fat in the metabolic syndrome. J Clin Endocrinol Metab 2007;92:3490-7.

41. Lee SS, Park SH, Kim HJ, et al. Non-invasive assessment of hepatic steatosis: prospective comparison of the accuracy of imaging examinations. J Hepatol 2010;52:579-85.

42. Cusi K, Sanyal AJ, Zhang S, et al. Non-alcoholic fatty liver disease (NAFLD) prevalence and its metabolic associations in patients with type 1 diabetes and type 2 diabetes. Diabetes Obes Metab 2017;19:1630-4.

43. Lai LL, Wan Yusoff WNI, Vethakkan SR, et al. Screening for non-alcoholic fatty liver disease in patients with type 2 diabetes mellitus using transient elastography. J Gastroenterol Hepatol 2019;34:1396-403.

44. Leite NC, Salles GF, Araujo AL, et al. Prevalence and associated factors of non-alcoholic fatty liver disease in patients with type-2 diabetes mellitus. Liver Int 2009;29:113-9.

45. Schwimmer JB, Newton KP, Awai HI, et al. Paediatric gastroenterology evaluation of overweight and obese children referred from primary care for suspected nonalcoholic fatty liver disease. Aliment Pharmacol Ther 2013;38:1267-7.

46. Hagström H, Talbäck M, Andreasson A et al. Ability of noninvasive scoring systems to identify individuals in the population at risk for severe liver disease. Gastroenterology 2020;158:200-14.

47. Tobari M, Hashimoto E, Taniai M, et al. Characteristics of non-alcoholic steatohepatitis among lean patients in Japan: Not uncommon and not always benign. J Gastroenterol Hepatol 2019;34:1404-10.

48. Leung JC, Loong TC, Wei JL, et al. Histological severity and clinical outcomes of nonalcoholic fatty liver disease in nonobese patients. Hepatology 2017;65:54-64.

49. Vilar-Gomez E, Chalasani N. Non-invasive assessment of non-alcoholic fatty liver disease: Clinical prediction rules 
and blood-based biomarkers. J Hepatol 2018;68:305-15.

50. Srivastava A, Gailer R, Tanwar S, et al. Prospective evaluation of a primary care referral pathway for patients with non-alcoholic fatty liver disease. J Hepatol 2019;71:371-8.

51. Harman DJ, Ryder SD, James MW, et al. Direct targeting of risk factors significantly increases the detection of liver cirrhosis in primary care: a cross-sectional diagnostic study utilising transient elastography. BMJ Open 2015;5:e007516.

52. Caussy C, Reeder SB, Sirlin CB, et al. Noninvasive, Quantitative Assessment of Liver Fat by MRI-PDFF as an Endpoint in NASH Trials. Hepatology 2018;68:763-2.

53. Yokoo T, Serai SD, Pirasteh A, et al. Linearity, Bias, and Precision of Hepatic Proton Density Fat Fraction Measurements by Using MR Imaging: A Meta-Analysis. Radiology 2018;286:486-98.

54. Kose S, Ersan G, Tatar B, et al. Evaluation of Percutaneous Liver Biopsy Complications in Patients with Chronic Viral Hepatitis. Eurasian J Med 2015;47:161-4.

55. Bedossa P, Poitou C, Veyrie N, et al. Histopathological algorithm and scoring system for evaluation of liver lesions in morbidly obese patients. Hepatology 2012;56:1751-9.

56. Serra JT, Mueller J, Teng H, et al. Prospective Comparison of Transient Elastography Using Two Different Devices: Performance of FibroScan and FibroTouch. Hepat Med 2020;12:41-8.

57. Liebig S, Stoeckmann N, Geier A, et al. Multicenter Validation Study of a Diagnostic Algorithm to Detect NASH and Fibrosis in NAFLD Patients With Low NAFLD Fibrosis Score or Liver Stiffness. Clin Transl Gastroenterol 2019;10:e00066.

58. Mayo R, Crespo J, Martínez-Arranz I, et al. Metabolomicbased noninvasive serum test to diagnose nonalcoholic steatohepatitis: Results from discovery and validation cohorts. Hepatol Commun 2018;2:807-20.

59. Roeb E. Fatty liver. In: Lammert F, Lynen Jansen P, Lerch M. editors. Weissbuch Gastroenterologie 2020/2021. Erkrankungen des Magen-Darm-Traktes, der Leber und der Bauchspeicheldrüse - Gegenwart und Zukunft. Berlin, Boston: De Gruyter, 2019:76-81.

60. Friedman S, Sanyal A, Goodman Z, et al. Efficacy and safety study of cenicriviroc for the treatment of nonalcoholic steatohepatitis in adult subjects with liver fibrosis: CENTAUR Phase 2b study design. Contemp Clin Trials 2016;47:356-65.

61. Sumida Y, Yoneda M. Current and future pharmacological therapies for NAFLD/NASH. J Gastroenterol
2018;53:362-76.

62. Sung KC, Wild SH, Byrne CD. Resolution of fatty liver and risk of incident diabetes. J Clin Endocrinol Metab 2013;98:3637-43.

63. Roeb E, Geier A. Nonalcoholic steatohepatitis (NASH) - current treatment recommendations and future developments. Z Gastroenterol 2019;57:508-17.

64. Sumida Y, Yoneda M, Ogawa Y, et al. Current and new pharmacotherapy options for non-alcoholic steatohepatitis. Expert Opin Pharmacother 2020;21:953-67.

65. Dougherty JA, Guirguis E, Thornby KA. A Systematic Review of Newer Antidiabetic Agents in the Treatment of Nonalcoholic Fatty Liver Disease. Ann Pharmacother 2021;55:65-79.

66. Cholankeril G, Wong RJ, Hu M, et al. Liver Transplantation for Nonalcoholic Steatohepatitis in the US: Temporal Trends and Outcomes. Dig Dis Sci 2017;62:2915-22.

67. Noureddin M, Vipani A, Bresee C, et al. NASH Leading Cause of Liver Transplant in Women: Updated Analysis of Indications For Liver Transplant and Ethnic and Gender Variances. Am J Gastroenterol 2018;113:1649-59.

68. Byrne CD, Targher G. EASL-EASD-EASO Clinical Practice Guidelines for the management of non-alcoholic fatty liver disease: is universal screening appropriate? Diabetologia 2016;59:1141-4.

69. HCC AASLD. Available online: https://aasldpubs. onlinelibrary.wiley.com/doi/pdf/10.1002/hep.29086 (6th May 2020)

70. HCC EASL. Available online: https://easl.eu/wpcontent/uploads/2018/10/EASL-CPG-Management-ofhepatocellular-carcinoma-.pdf (6th May 2020)

71. ECDC. Available online: https://www.ecdc.europa.eu/en/ immunisation-vaccines/EU-vaccination-schedules (4th May 2020)

72. STIKO. Available online: https://www.rki.de/DE/ Content/Kommissionen/STIKO/Empfehlungen/ Aktuelles/Impfkalender.html (4th May, 2020)

73. CDC. Available online: https://www.cdc.gov/vaccines/ (4th May 2020)

74. Sumida Y, Okanoue T, Nakajima A, et al. Phase 3 drug pipelines in the treatment of non-alcoholic steatohepatitis. Hepatol Res 2019;49:1256-62.

75. Neuschwander-Tetri BA, Loomba R, Sanyal AJ, et al. Farnesoid X nuclear receptor ligand obeticholic acid for non-cirrhotic, non-alcoholic steatohepatitis (FLINT): a multicentre, randomised, placebo-controlled trial. Lancet 2015;385:956-65. 
76. Younossi ZM, Ratziu V, Loomba R, et al. REGENERATE Study Investigators. Obeticholic acid for the treatment of non-alcoholic steatohepatitis: interim analysis from a multicentre, randomised, placebo-controlled phase 3 trial. Lancet 2019;394:2184-96.

77. Friedman SL, Ratziu V, Harrison SA, et al. A randomized, placebo-controlled trial of cenicriviroc for treatment of nonalcoholic steatohepatitis with fibrosis. Hepatology 2018;67:1754-67.

78. Sanyal AJ, Chalasani N, Kowdley KV, et al. Pioglitazone, vitamin $\mathrm{E}$, or placebo for nonalcoholic steatohepatitis. $\mathrm{N}$

Cite this article as: Roeb E. Non-alcoholic fatty liver diseases: current challenges and future directions. Ann Transl Med 2021;9(8):726. doi: 10.21037/atm-20-3760
Engl J Med 2010;362:1675-85.

79. Rubino F, Nathan DM, Eckel RH, et al. Metabolic Surgery in the Treatment Algorithm for Type 2 Diabetes: A Joint Statement by International Diabetes Organizations. Surg Obes Relat Dis 2016;12:1144-62.

80. S3 Obesity Surgery. Available online: https://www.awmf. org/uploads/tx_szleitlinien/088-0011_S3_ChirurgieAdipositas-metabolische-Erkrankungen_2018-02.pdf (6th May 2020) 